\title{
Research Paper: The Influence of Striatal Astrocyte Dysfunction on Locomotor Activity in Dopamine-depleted Rats
}

\author{
Dmitry Voronkov $^{1}$ (D), Alla Stavrovskaya ${ }^{1}$ (D), Artyom Olshanskiy' ${ }^{\text {(D) }}$, Anastasia Guschina ${ }^{1}$ (D), Rudolf Khudoerkov ${ }^{1}$ (D), Sergey Illarioshkin ${ }^{1}$ (i)
}

\begin{tabular}{|c|c|}
\hline $\begin{array}{l}\text { Us e your devive to ts san } \\
\text { and read the article online }\end{array}$ & Citation Voronkov, D., Stavrovskaya, A., Olshanskiy, A., Guschina, A., Khudoerkov, I \\
\hline 口ifiris & $\begin{array}{l}\text { ence of Striatal Astrocyte Dysfunction on Locomotor Activity in Dopamine-depleted Rats. Basic and Clinical Neuroscience, } \\
12(6), 767-776 . \text { http://dx.doi.org/10.32598/bcn.2021.1923.1 }\end{array}$ \\
\hline 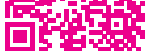 & dol http://dx.doi.org/10.32598/ben.2021.1923.1 \\
\hline
\end{tabular}

c) (i) (5)

Article info:

Received: 08 Jul 2019

First Revision: 23 Oct 2019

Accepted: 24 Jun 2020

Available Online: 01 Nov 2021

Keywords:

Astrocyte, 2-Aminoadipic Acid, Alpha-methyltyrosine, Corpus striatum, Motor activity

\begin{abstract}
AB S T RAC T
Introduction: Astrocyte dysfunction is the common pathology failing astrocyte-neuron interaction in neurological diseases, including Parkinson's Disease (PD). The present study aimed to evaluate the impacts of astrocytic dysfunction caused by striatal injections of selective glial toxin L-Aminoadipic Acid (L-AA) on the rats' locomotor activity in normal conditions and under alpha-methyl-p-tyrosine depletion of catecholamines synthesis.
\end{abstract}

Methods: Thirty-three male Wistar rats were used in the experiments. Intrastriatal L-AA injections $(100 \mu \mathrm{g})$ were performed into the right striatum. Alpha-methyl-p-tyrosine (a-MT, $100 \mathrm{mg} / \mathrm{kg}$, inhibitor of tyrosine hydroxylase) was intraperitoneally injected for catecholamine depletion. The animals were divided into 5 groups, as follows: 1 . L-AA treated $(\mathrm{n}=7), 2$. L-AA+a-MT treated $(n=5), 3$. Sham-operated $(n=7), 4$. Sham+a-MT treated $(n=5)$, 5. Intact control $(n=9)$. For assessing motor function, open field and beam walking tests were used on the third day after the operation. Neuronal and astrocyte markers (glial fibrillary acidic protein, glutamine synthetase, tyrosine hydroxylase, \& neuronal nuclear antigen) were examined in the striatum by immunohistochemistry.

Results: Administrating L-AA led to astrocytic degeneration in the striatum. No neuronal death and disruption of dopaminergic terminals were detected. L-AA and a-MT-treated animals' distance traveled was significantly $(\mathrm{P}=0.047)$ shorter than the Sham-operated group injected with a-MT. In the walking beam test, the number of unilateral paw slippings was significantly $(\mathrm{P}<0.01)$ higher in the L-AA-treated group than Sham-operated animals. Administrating a-MT alone and L-AA did not change rats' performance in walking beam tests.

Conclusion: Astrocyte ablation in dopamine depleted striatum resulted in reduced motor activity and asymmetrical gait disturbances. These findings demonstrated the role of astroglia in motor function regulation in the nigrostriatal system and suggest the possible association of glial dysfunction with motor dysfunction in PD.

* Corresponding Author:

Dmitry Voronkov, PhD.

Address: Research center of Neurology, Moscow, Russia.

Tel: +7 (9) 162169396

E-mail:voronkov@neurology.ru 


\section{Highlights}

- The local administration of gliotoxin L-aminoadipate in the striatum of rats causes astrocytic degeneration without affecting the neurons and nigrostriatal fibers.

- The failure of astrocyte-neuron coupling in the striatum leads to motor dysfunction such as gait disturbances and bradykinesia.

- The influence of astrocytic degeneration on behavior is preserved and enhanced in dopamine-depleted rats.

\section{Plain Language Summary}

Astrocytes are the nervous system's cells supporting the function of neurons. The failure of astrocyte-neuron interaction is observed in neurological diseases, including Parkinson's disease. We induced the aminoadipate-induced rat model of astrocytic dysfunction to evaluate the role of these cells in movement regulation. In our study, astrocytic dysfunction led to gait disturbances and impaired motor function. The results suggest a possible role of glial pathology in motor impairment in parkinsonism.

\section{Introduction}

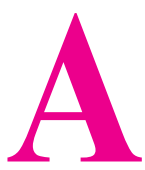

strocytic dysfunction has been implicated in several neurological disorders epilepsy, primary and chronic depressive disorder, and neurodegenerative disorders, such as frontotemporal dementia, Alzheimer's Disease (AD), Huntington, and Parkinson's Diseases (PD) (Banasr \& Duman, 2008; Halliday \& Stevens, 2011; Verkhratsky, Parpura, Pekna, Pekny, \& Sofroniew, 2014). The degeneration of astrocytes associated with chronic depressive disorder (Smiałowska, Szewczyk, Woźniak, Wawrzak-Wleciał, \& Domin, 2013) and frontotemporal dementia (Broe, Kril, \& Halliday, 2004). Contrarily, an excessive pro-inflammatory astrocyte reaction is assumed among the mechanisms of neuronal damage in PD and AD (Liddelow \& Barres, 2017; Verkhratsky et al., 2014). Mutation of the Glial Fibrillary Acidic Protein (GFAP) gene, leading to disruption of the astrocyte cytoskeleton, led to abnormal GFAP accumulation, neuroinflammation, and leukodystrophy in Alexander's disease (Olabarria \& Goldman, 2017).

Astrocytes regulate numerous neuronal functions, such as synaptogenesis, axon pruning, neuronal metabolism, and neurotransmission (Sofroniew \& Vinters, 2010). Activation of astroglia and gliosis is the universal response of the nervous tissue on the lesion. Activated astroglia plays ambivalent pro-inflammatory and neuroprotective roles, producing signal molecules and interacting with microglia. Neuroinflammation or ischemia leads to the formation of phenotypically different A1 (pro-inflammatory) and A2 (anti-inflammatory) states of activated astrocytes, respectively (Anderson, Ao, \& Sofroniew, 2014; Liddelow \& Barres, 2017).

Astrocytes take part in tissue remodeling during neurodegeneration. These cells regulate axonal growth, produce extracellular matrix molecules, and remove cell debris (Sofroniew \& Vinters, 2010). Furthermore, astrocytes provide a compensatory regulation of neurotransmission, interacting with synapses and maintaining the concentration of extracellular glutamate, gammaAminobutyric acid (GABA), and dopamine (Sofroniew \& Vinters, 2010).

In $\mathrm{PD}$, astrocytes reactions include but are not limited to the pro-inflammatory response. Astrocytes, like neurons, express genes associated with autosomal recessive forms of PD (e.g. Parkin, PINK-1 and DJ-1, LRRK2) (Halliday $\&$ Stevens, 2011). Moreover, the mutations of these genes lead to astrocytic dysfunction (Mullett, Di Maio, Greenamyre, \& Hinkle, 2013; Zhao et al., 2018). In Lewy body dementia, PD, and several tauopathies, abnormal protein inclusions were found in neurons and astrocytes (Kovacs, 2015; Rostami et al., 2017). In PD, astrocytes accumulate toxic forms of $\alpha$-synuclein and play a role in their propagation in the Central Nervous System (CNS) (Cavaliere et al., 2017; Lindström et al., 2017).

Therapeutic potential and the possibilities of the pharmacological regulation of the functions of astrocytes and the "management" of glial reaction in trauma, stroke, and neurodegenerative diseases are widely discussed (Liddelow \& Barres, 2017). The neuroprotective effect 
of astrocyte transplantation and their co-transplantation with neurons (Nicaise, Mitrecic, Falnikar, \& Lepore, 2015; Proschel, Stripay, Shih, Munger, \& Noble, 2014; Song et al., 2018) was presented on the models of neurodegenerative diseases. Moreover, astrocyte transdifferentiation towards neuronal phenotype occurs in non-neurogenic brain structures under pathological conditions. The formation of neurons from striatal astrocytes was shown on the model of ischemic brain infarction in rodents (Duan et al., 2015).

The role of astroglia in processing information in neural networks remains unclear (Fiacco \& McCarthy, 2018; Savtchouk \& Volterra, 2018). The astrocyte-neuron interaction and its effect on neuronal activity have been found both in vivo and in vitro (Sofroniew \& Vinters, 2010). The specificity of astrocyte distribution and their molecular heterogeneity depends on the brain region and is closely related to the participation of glia in the synaptic transmission reflecting its interaction with neurons (Emsley \& Macklis, 2006). Each astrocytic process is located in an individual domain in the neocortex and hippocampus, forming a neuro-glio-vascular structural unit (Sofroniew \& Vinters, 2010). It is known that gap junctions couple astrocytes; however, whether they create functional networks in different brain regions remains undiscovered (Emsley \& Macklis, 2006; Fiacco \& McCarthy, 2018; Savtchouk \& Volterra, 2018).

The possibility to impact astroglial function is limited in the experiment. Unlike neurons, a small number of selective models of astrocytic degeneration have been developed. The transgenic animal model with targeted depletion of GFAP-positive astroglia (glial fibrillary acidic protein) (Jäkel \& Dimou, 2017) and vimentin and GFAP knock-out mice, characterized by reduced glial reactivity (Laterza et al., 2018; Wilhelmsson et al., 2004). To date, only fluorocitrate and L-aminoadipic acid are used for the selective chemical ablation of astrocytes (Willoughby et al., 2003, Khurgel, Koo, \& Ivy, 1996). Fluorocitrate is an aconitase inhibitor, and being captured by glial cells suppresses the metabolism of astrocytes, leading to their damage (Kuter, Olech, \& Głowacka, 2018; Voloboueva et al., 2007). However, fluorocitrate exhibits a nonspecific toxic action (Fonnum, Johnsen, \& Hassel, 1997). The structural analogue of glutamate, L-isoform of alpha-Aminoadpic Acid (L-AA), is selectively toxic to astrocytes in vitro and in vivo. Furthermore, the toxin is captured by Na-dependent glutamate transporters. It also causes a decrease in protein synthesis and astrocyte apoptosis. However, the mechanism of L-AA action remains unclear (Nishimura, Santos,
Fu, \& Dwyer, 2000; Smiałowska et al., 2013). Electron microscopy studies indicated that single injections of L-AA into the prefrontal cortex, striatum, and amygdala result in glial cell death without affecting neurons directly (Billet, Costentin, \& Dourmap, 2007; Saffran \& Crutcher, 1987; Smiałowska et al., 2013; Takada \& Hattori, 1986). The restoration of immunoreactivity to GFAP occurs 7-10 days after administration due to the proliferation and migration of astrocytes toward the area of damage (Khurgel et al., 1996). L-AA administered in the cortex and amygdala leads to depressive-like behavior in rats (Banasr \& Duman, 2008; Smiałowska et al., 2013). At the same time, there are reports on the absence of the gliotoxic action of L-AA administered in the striatum and hippocampus (Saffran \& Crutcher, 1987).

Thus, multiple studies indicated astroglia's involvement in modulating striatum neurons' activity under normal and pathological conditions (Chai et al., 2017; Dvorzhak, Melnick, \& Grantyn, 2018; Villalba, Mathai, \& Smith, 2015). Although astrocytes contain dopamine metabolizing enzymes monoamine oxidase and catechol-O-methyltransferase, the role of glia in modulating the functions of the nigrostriatal dopaminergic system remains debatable (Jennings \& Rusnakov 2016). Moreover, the association between glial dysfunction and the development of extrapyramidal disorders remains undiscovered. Assessing motor dysfunction on a model with a combination of dopamine synthesis inhibition (by alpha-methyl-p-tyrosine, a-MT) and astrocytes ablation will allow evaluating the role of astroglia in the striatum.

This study aimed to evaluate the impact of astrocytic dysfunction caused by striatal injections of selective glial toxin L-Aminoadipic Acid (L-AA) on the rats' locomotor activity in normal conditions and under alpha-methyl-p-tyrosine depletion of catecholamines synthesis.

\section{Methods}

Male Wistar rats (3.5-4 months old) were kept under a $12 \mathrm{~h}$ dark/light cycle, with free access to food and water. All experiments were conducted according to the guide for the care and use of laboratory animals (National Institutes of Health Newsletter No. 80-23, revised 1996). This study was approved by the Ethics Committee of the Research Centre of Neurology (Moscow, Russia), protocol No. 2-5 / 19 of 02.20.19.

Intrastriatum injections under stereotaxic surgery were performed on 24 rats. Half of them were administered Laminoadipic acid (L-AA, $100 \mu \mathrm{g}$ ); other rats were Shamoperated $(n=12)$. On the third day after stereotaxic opera- 
tions and one hour before locomotor activity testing, part of the L-AA-treated group $(n=5)$ and 5 animals from the Sham-operated group were injected with alpha-methyl-ptyrosine (a-MT, $100 \mathrm{mg} / \mathrm{kg}$, IP, Sigma, USA), a tyrosine hydroxylase inhibitor (Watanabe et al., 2005), to establish a dopamine-depletion model. The remaining 7 animals in each group received saline injections instead of a-MT.

The experimental groups, therefore, consisted (Figure 1) of group 1, L-AA treated, Intraperitoneal (IP) received saline $(n=7)$; group 2, L-AA treated and IP received a-MT $(n=5)$; group 3, Sham-operated, IP received saline $(\mathrm{n}=7)$; Group 4, Sham-operated, IP received a-MT $(n=5)$. The control groups received no interventions.

\section{Stereotactic surgery}

The intrastriatal injections of L-AA were performed to induce astrocytic degeneration. Injections were performed under Zoletil-100 (Vibrac Sante Animale, France) and xylanite anesthesia ( $3 \mathrm{mg} / 100 \mathrm{~g}$ and $3 \mathrm{mg} / \mathrm{kg}$, IM, respectively). Atropine $(0.04 \mathrm{mg} / \mathrm{kg}, \mathrm{sc})$ was administered 10-15 min before xylanite injection. This measure aimed to prevent bronchial and laryngospasm, bradycardia, and cardiac arrest by weakening the vagal effects.

The stock solution of L-AA (Acros Organics, Belgium) was dissolved in $1 \mathrm{M} \mathrm{HCl}$ at a concentration of $120 \mu \mathrm{g} /$ $\mu \mathrm{L}$. The solution used for administration was prepared in Phosphate-Buffered Saline (PBS), adjusted with 1M $\mathrm{NaOH}$ to $\mathrm{pH}=7.3$, and diluted to the final L-AA concentration of $20 \mu \mathrm{g} / \mu \mathrm{L}$ (Khurgel et al., 1996). Anesthetized animals were placed in the dual-arm stereotaxic unit (Stoelting Co., USA). L-AA solution $(5 \mu \mathrm{L})$ was injected to the right striatum unilaterally at the following coordinates: $\mathrm{AP}=1.5 ; \mathrm{L}=2.5 ; \mathrm{V}=4,8$ (Paxinos \& Watson, 2007). The same volume of PBS was injected into the left hemisphere. The Sham-operated animals received injections of $5 \mu \mathrm{L}$ of PBS, bilaterally.

\section{Locomotor activity tests}

Behavioral testing was carried out 72 hours after the administration of L-AA. This period was chosen according to previous reports on the maximum decrease of astrocytes density on the days 2-4 after L-AA administration (Khurgel et al., 1996). The locomotor activity of experimental rats was evaluated with an open field and beam walking test.

Locomotor test in the open field for 3 min, including the total number of crossed squares for $3 \mathrm{~min}$, was evaluated in the open field arena $(75 \mathrm{~cm} \mathrm{~L} \mathrm{x75} \mathrm{cm} \mathrm{W} \mathrm{x40} \mathrm{cm}$
$\mathrm{H})$ divided into 25 equal squares. The control group in this test consisted of 9 animals.

Beam walking test apparatus consists of a ledged tapered beam 1 meter long resting $70 \mathrm{~cm}$ above the floor (Schallert, Woodlee, \& Fleming, 2002). Side ledges extend to the sides underneath the beam. The width of the walking bar is 2 to $2.5 \mathrm{~cm}$; height is $1 \mathrm{~cm}$. All along the track, below its level, on both sides, $2 \mathrm{~cm}$ tabs allow the animal to put a weakened front- or hindlimb in case of slipping so as not to fall off the track. There is a shelter at the narrow end of the beam with a removable lid. The animals passed along the upper bar and counted the number of complete steps and the percentage of the fore and hind limbs' slips (foot faults). The control group in this test consisted of 5 animals.

\section{Immunohistochemistry}

Immediately after conducting the behavioral tests, randomly selected L-AA-treated $(\mathrm{n}=5)$ and Sham-operated $(n=5)$ animals were decapitated by guillotine following xylanite anesthesia. The extracted brain was fixed in PBS buffered 4\% formalin for 24 hours. Then, the study samples were soaked with $30 \%$ sucrose and O.C.T. compound (TissueTek, Netherlands) and cut on a series of frozen frontal sections (12- $\mu \mathrm{m}$ thick). For an immunohistochemical study, heat-induced epitope retrieval was carried out in citrate buffer $(1 \mathrm{M}, \mathrm{pH}=6.0)$ with $0.1 \%$ Tween-80 for $20 \mathrm{~min}$ in a steamer. Monoclonal mouse antibodies (Sigma) to GFAP (gliofibrillary protein, 1:150, Sigma), rabbit anti-NeuN (neuronal nuclear antigen, 1:800, Abcam), rabbit anti-tyrosine hydroxylase (1:800, Sigma), or rabbit anti-glutamine synthetase (1:1000, Sigma) were used. Sections were incubated with antibodies overnight in a humidity chamber. Appropriate secondary antibodies (Sigma) to rabbit and mouse immunoglobulins labeled with CF488 or CF555 fluorochromes were applied for visualization. PBS with $0,1 \%$ Triton X-100 was used at each step for rinsing slides. The slides were coverslipped with Fluorshield (SigmaAldrich) medium.

ImageJ software performed the counting of neurons and mean intensity measurement on the area of interest using the images acquired with Nikon Eclipse microscope (x40). At least 25 fields of view within 5-8 sections of each animal were taken for evaluation.

The obtained data were analyzed using one-way Analysis of Variance (ANOVA) and Newman-Keuls Posthoc test (to compare the differences between the study 


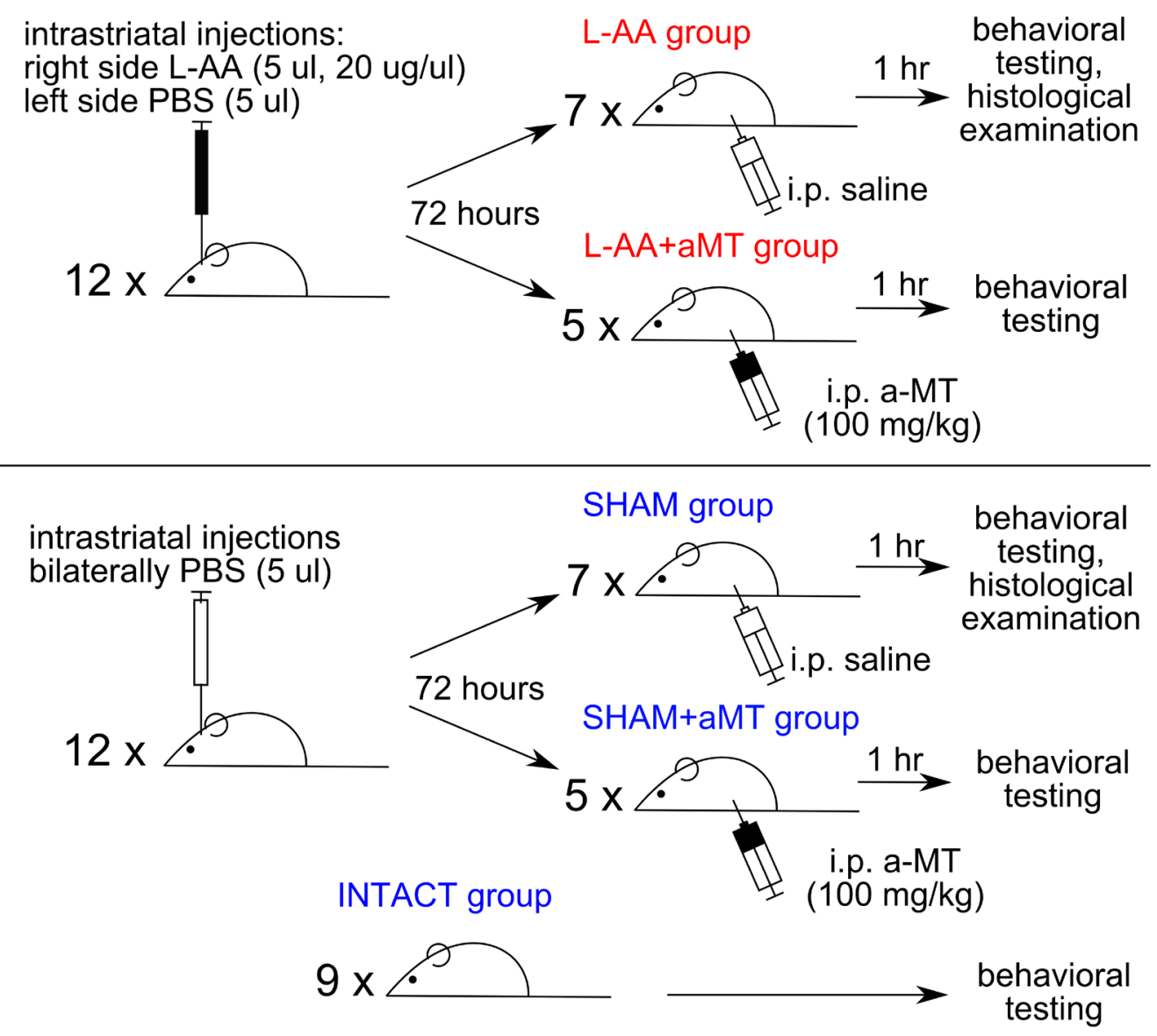

Figure 1. The experimental design

NEUR SCIENCE

groups) at the significance level of $\mathrm{P}<0.05$. Furthermore, all results are presented as Mean $\pm \mathrm{SEM}$.

\section{Results}

The histological examination of L-AA induced lesion

Reduced GFAP-reactivity, preserved NeuN-positive neurons, and tyrosine hydroxylase expression were observed in a wide injection area in the caudate nucleus on a 3-rd day after L-AA administration (Figure 2). In the injection area, astrocytic degeneration and the significant decrease of GFAP and glutamine synthetase staining were found (Figure 2). On the side of L-AA injection, glutamine synthetase was found only in the cytoplasm of ovoid glial cells considered oligodendroglia (Anlauf \& Derouiche, 2013) or immature astrocytes. Hypertrophic activated astrocytes surrounded the area of the lesion. Elevated GFAP expression was found in activated astrocytes near the needle track on the contralateral side. Slight damage of tissue was found around the track.
The mean intensity of GFAP staining in the area surrounding the needle track $(800 \mu \mathrm{m})$ in L-AA-injected striatum was $83 \%$ lower compared to that on the contralateral side (Mann-Witney Test; $\mathrm{P}<0.05$ ).

No difference in neuronal density estimated by the number of NeuN-positive cells was found between injected and non-injected sides. These results indicate that the effect of L-AA was astrocyte-selective (Figure 3). Furthermore, no differences between the intensity of tyrosine hydroxylase staining and morphology of dopaminergic nigrostriatal fibers in the area of L-AA injection and the intact regions of the striatum were found. Thus, the results of the immunohistochemical study demonstrated that GFAP-positive astroglia was disrupted in the broad area surrounding the injection site on the 3rd day after the L-AA administration. However, no degenerative changes of the striatal neurons or nigrostriatal dopaminergic terminals were detected. 


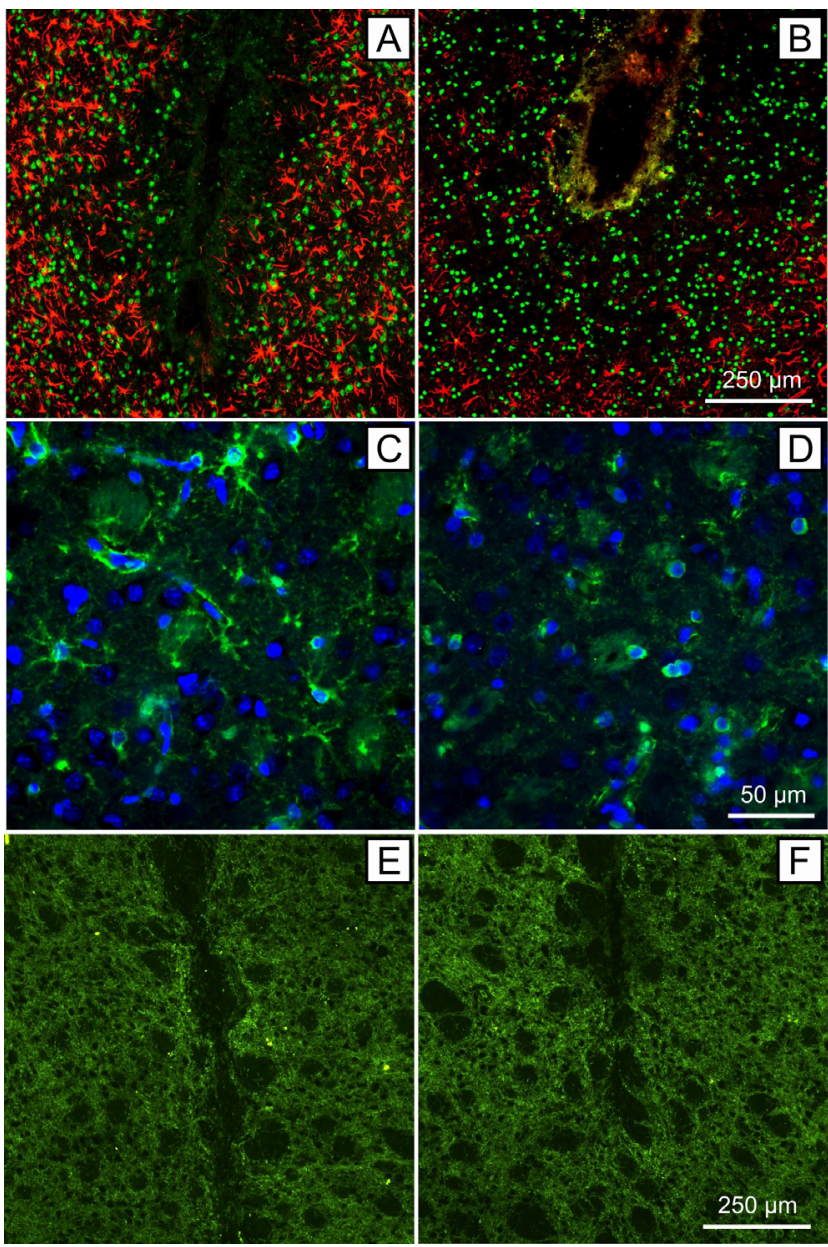

NEUR SCIENCE

Figure 2. The immunofluorescence staining of glial and neuronal markers in the striatum of L-AA-treated rats (72 hours after stereotaxic injection)

Left column-control, injected with PBS; Right column - L-AA-injected.

A, B: GFAP (red) and NeuN (green); C, D: Glutamine synthetase (green), DAPI counterstain (blue); E, F: Tyrosine hydroxylase (green) Immunofluorescence staining.

The behavioral effects of L-AA in control and dopamine-depleted rats

Significant differences between experimental groups (Figure 4 ) in the open field test were found $\left(\mathrm{F}_{4,28}=17.24\right.$, $\mathrm{P}<0.0001)$. The locomotor activity of Sham-operated rats was significantly lower than intact animals $(\mathrm{P}=0.02)$. To some extent, a decrease in locomotor activity in the Sham-operated group resulted from a shortened period between stereotaxic operation and testing (Stavrovskaya, Yamshchikova, Ol'shanskiy, Konovalova, \& Illarioshkin, 2017). Nevertheless, the post-hoc NewmanKeuls test revealed no significant differences between Sham-operated animals and the L-AA-treated group $(\mathrm{P}=0.09)$. The distance traveled by animals injected with a-MT was significantly shorter in both L-AA-treated
$(\mathrm{P}=0.02)$ and Sham-operated $(\mathrm{P}=0.04)$ groups than in ones received no a-MT. These findings are consistent with those reported by Watanabe et al. in experiments on a-MT-induced dopamine depletion models (Watanabe et al., 2005). L-AA and a-MT-treated animals' distance traveled was significantly $(\mathrm{P}=0.047)$ shorter than the Sham-operated group injected with a-MT only (Figure 4). Thus, results indicate that the degeneration of striatal astrocytes caused no dramatic changes in the activity of animals with the intact nigrostriatal system in an open field test. However, L-AA significantly affected the activity in dopamine-depleted rats.

In the walking beam test, the impairment of locomotor function and the hemiparetic-like effect were found in L-AA- treated animals (Figure 5). One-way ANOVA 

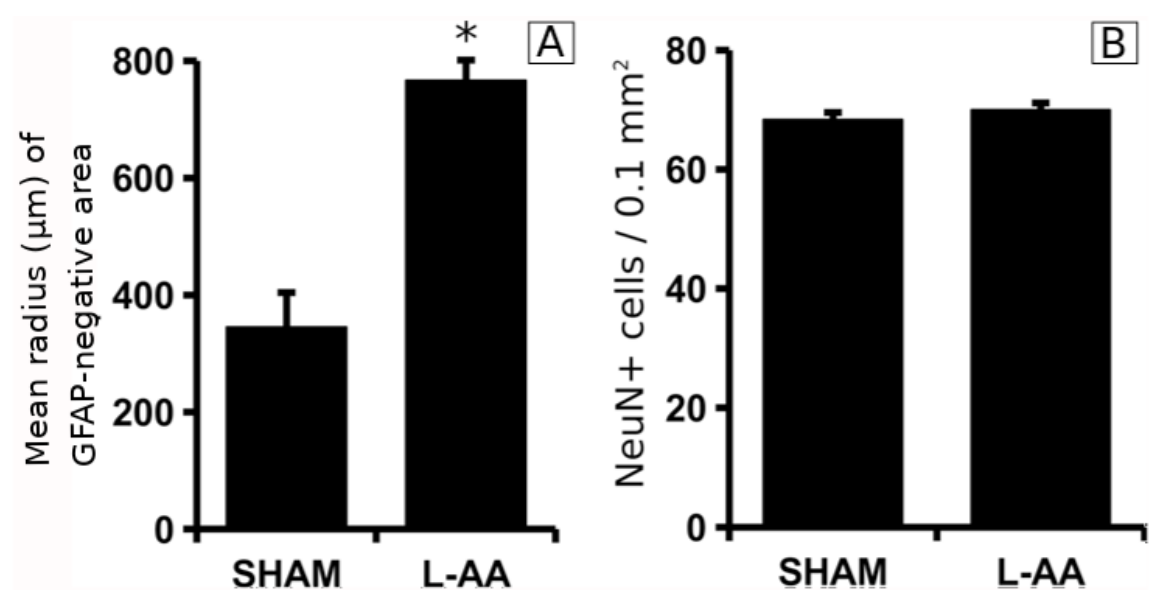

NEUROSCIENCE

Figure 3. Morphological changes in striatum 72 hours after stereotaxic injections of L-Amino-Adipic acid (L-AA), compared to the Sham-operated rats

A: Mean radius $(\mu \mathrm{m})$ of GFAP+ astrocytes depleted area adjacent to needle track.

B: Neurons ( $\mathrm{NeuN}^{+}$cells) count in $800 \mu \mathrm{m}$ width area around needle track.

${ }^{*} \mathrm{P}<0.05$ Mann-Witney test. The results are represented as Mean \pm SEM.

data indicated the significant impact of L-AA on the number of paw slippings on the side contralateral to injection (left) in groups $\left(\mathrm{F}_{4,24}=10.58, \mathrm{P}<0.0001\right)$, and no group difference in foot faults on the right side. No significant differences were found between intact and Sham-operated animals. The a-MT administration in Sham-operated animals did not affect the number of slippings. The number of paw slippings on the left side was significantly $(\mathrm{P}<0.01)$ higher in the L-AA-treated group than Sham-operated animals. The number of paw slippings in L-AA treated animals after a-MT administration was significantly $(\mathrm{P}=0.013)$ higher than the Sham-operated group after a-MT administration. Thus, a-MT did not increase the effect of 1-AA in the walking beam test, which was different from the results in an open field test.

\section{Discussion}

The revealed L-AA effect on locomotion was associated with astrocyte degeneration. The preservation of neurons and nigro-striatal terminals was morphologically verified. However, the L-AA administration provoked the

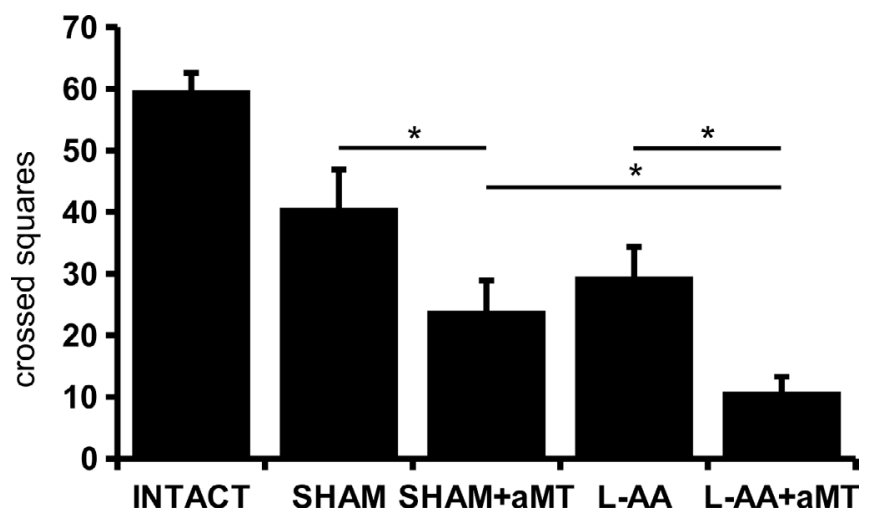

NEUR SCIENCE

Figure 4. The effect of alpha-methyl-p-tyrosine (aMT) treatment and L-amino-Adipic Acid (L-AA) intrastriatal injections on locomotor activity in the open field test

Experimental groups: INTACT-intact rats; SHAM-sham-operated rats; SHAM+aMT - Sham-operated rats injected with aMT; L-AA -L-AA-treated rats; LAA-aMT - L-AA-treated rats injected with aMT.

${ }^{*} \mathrm{P}<0.05$ Newman-Keuls Post hoc-test. Intact, the rats' group was significantly different from all groups (asterisk not shown). The results are represented as Mean \pm SEM. 


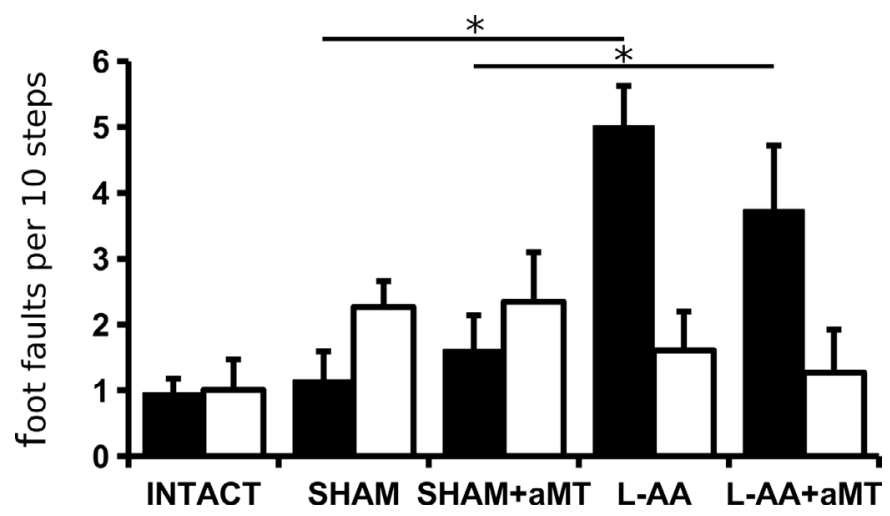

Figure 5. The asymmetrical gait disturbance of L-AA-treated rats in the beam walking test

The designation of experimental groups is the same as in Figure 3.

Black bars-left limbs (contralateral to L-AA intrastriatal injection), White bars-right limbs. ${ }^{*}<<0.05$ Newman-Keuls Post hoc-test. The results are represented as Mean \pm SEM.

degeneration of astrocytes and significant glial reactivity surrounding the glial toxin injection area. Therefore, the L-AA-mediated model should be considered as the model of astrocytic dysfunction and not as an astrocytic "shutdown". In general, a glial response to L-AA injection corresponds to changes observed in a wide range of neurodegenerative pathologies, characterized by the coexistence of astrocytic degeneration, impairment of glianeuron coupling, and gliosis (Verkhratsky et al., 2014).

An increase in the number of GFAP-positive astrocytes after lesion of nigrostriatal dopaminergic terminals in the striatum was demonstrated on various models of Parkinson's disease. Thus, MPTP administration induces the expansion of the area of contact of astrocytic processes with synapses in the striatum, i.e., associated with regulating glutamatergic neurotransmission and elevated uptake of extracellular glutamate (Villalba et al., 2015). Moreover, the fluorocitrate-induced nigral astrocytes damage decelerates the recovery of 6-OHDA-induced locomotor impairments (Kuter et al., 2018). These findings reflect the neuroprotective and compensatory role of astroglia activation. Consequently, respecting nigrostriatal system dysfunction, astrocytes provide a compensatory response, and astrocytic degeneration or dysfunction aggravates the neurodegenerative process in PD. Astrocytes regulate basal ganglia functions and serve as modulators of volume neurotransmission and dynamic regulators of the strength and kinetics of synaptic activity (Dvorzhak et al., 2018; Villalba et al., 2015). Astrocytes are involved in the metabolism of glutamate, GABA, and dopamine (Jennings \& Rusakov, 2016), i.e., the primary mediators controlling the activity of striatal medium spiny projection neurons. Neurotransmitter imbalance in the caudate nucleus, caused by astrocytic death after
L-AA administration, probably led to locomotion dysfunction. Interestingly, L-AA-treated rats demonstrated gait asymmetry in the beam walk test, which could be due to improper placement of limbs ipsilateral to lesion causing slippering to the contralateral side.

As reported by Billet, striatal L-AA infusion causes an increase and then subsequent decrease of glutamate levels depending on the post-operation period (Billet et al., 2007). That can result from disruption of glutamate metabolism in glial cells and an alteration of neuronal pools of glutamate. Despite the reported absence of dopamine / DOPAC content variations after L-AA infusion, we suppose that enhanced severity of bradykinesia induced by L-AA and a-MT combination treatment reflects astrocytes' role in modulation dopaminergic neurotransmission (Billet et al., 2007). Astrocytes express catecholO-methyl transferase and monoamine oxidase, and their damage leads to local elevation of extrasynaptic dopamine levels. The disruption of astrocyte-neuron coupling in the L-AA model causes an increase in the extracellular glutamate content, a failure of dopaminergic modulation in the cortico-striatal pathway, and an imbalance of inhibitory and excitation actions in the Nigro-strio-nigral loop.

\section{Conclusion}

Our study data demonstrated that the L-AA astrocyte ablation model is a perspective approach for investigating astroglial function in the pathogenesis of neurodegenerative diseases. L-AA-induced striatal astrocyte degeneration affected the locomotion of rats. The results emphasize the regulatory role of astrocytes in the nigrostriatal system and reveal the possible association between glial dysfunction and motor dysfunction in PD 


\section{Ethical Considerations}

\section{Compliance with ethical guidelines}

All experiments were conducted according to the guide for the care and use of laboratory animals (National Institutes of Health Newsletter No. 80-23, revised 1996). This study was approved by the Ethics Committee of the Research Centre of Neurology, Moscow, Russia (Protocol No.: 2-5/19 of 02.20.19).

\section{Funding}

This research did not receive any grant from funding agencies in the public, commercial, or non-profit sectors.

\section{Authors' contributions}

Conceptualization and supervision: Sergey Illarioshkin and Rudolf Khudoerkov; Methodology: Dmitry Voronkov and Alla Stavrovskaya; Data collection and data analysis: Dmitry Voronkov and Alla Stavrovskaya, Artyom Olshanskiy, and Anastasia Guschina; Writing - original draft and writing - review and editing: All authors.

\section{Conflict of interest}

All authors have no conflict of interest to disclose

\section{Refrences}

Anderson, M. A., Ao, Y., \& Sofroniew, M. V. (2014). Heterogeneity of reactive astrocytes. Neuroscience Letters, 565, 23-29. [DOI:10.1016/j.neulet.2013.12.030] [PMID] [PMCID]

Anlauf, E., \& Derouiche, A. (2013). Glutamine synthetase as an astrocytic marker: Its Cell Type and Vesicle Localization. Frontiers in Endocrinology, 4, 144. [DOI:10.3389/fendo.2013.00144] [PMID] [PMCID]

Banasr, M., \& Duman, R. S. (2008). Glial loss in the prefrontal cortex is sufficient to induce depressive-like behaviors. Biological Psychiatry, 64(10), 863-870. [DOI:10.1016/j.biopsych.2008.06.008] [PMID] [PMCID]

Billet, F., Costentin, J., \& Dourmap, N. (2007). Influence of glial cells in the dopamine releasing effect resulting from the stimulation of striatal $\delta$-opioid receptors. Neuroscience, 150(1), 131-143. [DOI:10.1016/j.neuroscience.2007.09.004] [PMID]

Broe, M., Kril, J., \& Halliday, G. M. (2004). Astrocytic degeneration relates to the severity of disease in frontotemporal dementia. Brain, 127(Pt 10), 2214-2220. [DOI:10.1093/brain/ awh250] [PMID]
Cavaliere, F., Cerf, L., Dehay, B., Ramos-Gonzalez, P., De Giorgi, F., \& Bourdenx, M., et al. (2017). In vitro a-synuclein neurotoxicity and spreading among neurons and astrocytes using Lewy body extracts from Parkinson disease brains. Neurobiology of Disease, 103, 101-112. [DOI:10.1016/j. nbd.2017.04.011] [PMID]

Chai, H., Diaz-Castro, B., Shigetomi, E., Monte, E., Octeau, J. C., \& Yu, X., et al. (2017). Neural circuit-specialized astrocytes: Transcriptomic, proteomic, morphological, and functional evidence. Neuron, 95(3), 531-549. e9. [DOI:10.1016/j. neuron.2017.06.029] [PMID] [PMCID]

Duan, C. L., Liu, C. W., Shen, S. W., Yu, Z., Mo, J. L. \& Chen, X. H., et al. (2015). Striatal astrocytes transdifferentiate into functional mature neurons following ischemic brain injury. Glia, 63(9), 1660-1670. [DOI:10.1002/glia.22837] [PMID] [PMCID]

Dvorzhak, A., Melnick, I., \& Grantyn, R. (2018). Astrocytes and presynaptic plasticity in the striatum: Evidence and unanswered questions. Brain Research Bulletin, 136, 17-25. [DOI:10.1016/j.brainresbull.2017.01.001] [PMID]

Emsley, J. G., \& Macklis, J. D. (2006). Astroglial heterogeneity closely reflects the neuronal-defined anatomy of the adult murine CNS. Neuron Glia Biology, 2(3), 175-186. [DOI:10.1017/S1740925X06000202] [PMID] [PMCID]

Fiacco, T. A., \& McCarthy, K. D. (2018). Multiple lines of evidence indicate that gliotransmission does not occur under physiological conditions. Journal of Neuroscience, 38(1), 3-13. [DOI:10.1523/JNEUROSCI.0016-17.2017] [PMID] [PMCID]

Fonnum, F., Johnsen, A., \& Hassel, B. (1997). Use of fluorocitrate and fluoroacetate in the study of brain metabolism. Glia, 21(1), 106-113. [DOI:10.1002/(SICI)10981136(199709)21:1\%3C106::AID-GLIA12\%3E3.0.CO;2-W] [PMID]

Halliday, G. M., \& Stevens, C. H. (2011). Glia: Initiators and progressors of pathology in parkinson's disease. Movement disorders: Official Journal of the Movement Disorder Society, 26(1), 6-17. [DOI:10.1002/mds.23455] [PMID]

Jäkel, S. \& Dimou, L. (2017). Glial cells and their function in the adult brain: A journey through the history of their ablation. Frontiers in Cellular Neuroscience, 11, 24. [DOI:10.3389/ fncel.2017.00024] [PMID] [PMCID]

Jennings, A., \& Rusakov, D. A. (2016). Do astrocytes respond to dopamine? Opera Medica et Physiologica, 2(1), 34-43. [DOI:10.20388/OMP2016.001.0017]

Khurgel, M., Koo, A. C., \& Ivy, G. O. (1996). Selective ablation of astrocytes by intracerebral injections of alpha-aminoadipate. Glia, 16(4), 351-358. [DOI:10.1002/(SICI)10981136(199604)16:43.0.CO;2-2] [PMID]

Kovacs, G. G. (2015). Invited review: Neuropathology of tauopathies: Principles and practice. Neuropathology and Applied Neurobiology, 41(1), 3-23. [DOI:10.1111/nan.12208] [PMID]

Kuter, K., Olech, Ł., \& Głowacka, U. (2018). Prolonged dysfunction of astrocytes and activation of microglia accelerate degeneration of dopaminergic neurons in the rat substantia nigra and block compensation of early motor dysfunction induced by 6-OHDA. Molecular Neurobiology, 55(4), 30493066. [DOI:10.1007/s12035-017-0529-z] [PMID] [PMCID] 
Laterza, C., Uoshima, N., Tornero, D., Wilhelmsson, U., Stokowska, A., \& Ge, R., et al. (2018). Attenuation of reactive gliosis in stroke-injured mouse brain does not affect neurogenesis from grafted human iPSC-derived neural progenitors. PloS One, 13(2), e0192118. [DOI:10.1371/journal. pone.0192118] [PMID] [PMCID]

Liddelow, S. A., \& Barres, B. A. (2017). Reactive Astrocytes: Production, function, and therapeutic potential. Immunity, 46(6), 957-967. [DOI:10.1016/j.immuni.2017.06.006] [PMID]

Lindström, V., Gustafsson, G., Sanders, L. H., Howlett, E. H., Sigvardson, J., \& Kasrayan, A., et al. (2017). Extensive uptake of a-synuclein oligomers in astrocytes results in sustained intracellular deposits and mitochondrial damage. Molecular and Cellular Neurosciences, 82, 143-156. [DOI:10.1016/j. mcn.2017.04.009] [PMID]

Mullett, S. J., Di Maio, R., Greenamyre, J. T., \& Hinkle, D. A (2013). DJ-1 expression modulates astrocyte-mediated protection against neuronal oxidative stress. Journal of Molecular Neuroscience, 49(3), 507-511. [DOI:10.1007/s12031-012-9904-4] [PMID] [PMCID]

Nicaise, C., Mitrecic, D., Falnikar, A., \& Lepore, A. C. (2015). Transplantation of stem cell-derived astrocytes for the treatment of amyotrophic lateral sclerosis and spinal cord injury. World Journal of Stem Cells, 7(2), 380-398. [DOI:10.4252/wjsc. v7.i2.380] [PMID] [PMCID]

Nishimura, R. N., Santos, D., Fu, S. T., \& Dwyer, B. E. (2000) Induction of cell death by L-alpha-aminoadipic acid exposure in cultured rat astrocytes: Relationship to protein synthesis. Neurotoxicology, 21(3), 313-320. [PMID]

Olabarria, M., \& Goldman, J. E. (2017). Disorders of astrocytes: Alexander disease as a model. Annual Review of Pathology: Mechanisms of Disease, 12, 131-152. [DOI:10.1146/annurevpathol-052016-100218] [PMID]

Paxinos, G., \& Watson, C. (2007). The rat brain in stereotaxic coordinates in stereotaxic coordinates. Amsterdam: Elsevier. https:/ / books.google.com/books?id=3EPQj\&dq=The+RatDEAE

Proschel, C., Stripay, J. L., Shih, C. H., Munger, J. C., \& Noble, M. D. (2014). Delayed transplantation of precursor cell-derived astrocytes provides multiple benefits in a rat model of parkinsons. EMBO Molecular Medicine, 6(4), 504-518. [DOI:10.1002/ emmm.201302878] [PMID] [PMCID]

Rostami, J., Holmqvist, S., Lindström, V., Sigvardson, J., Westermark, G. T., \& Ingelsson, M., et al. (2017). Human astrocytes transfer aggregated alpha-synuclein via tunneling nanotubes. Journal of Neuroscience, 37(49), 11835-11853. [DOI:10.1523/ JNEUROSCI.0983-17.2017] [PMID] [PMCID]

Saffran, B. N., \& Crutcher, K. A. (1987). Putative gliotoxin, alpha-aminoadipic acid, fails to kill hippocampal astrocytes in vivo. Neuroscience Letters, 81(1-2), 215-220. [DOI:10.1016/03043940(87)91001-9] [PMID]

Savtchouk, I., \& Volterra, A. (2018). Gliotransmission: Beyond black-and-white. Journal of Neuroscience, 38(1), 14-25. [DOI:10.1523/JNEUROSCI.0017-17.2017] [PMID] [PMCID]

Schallert, T., Woodlee, M. T. \& Fleming, S. M. (2000). Disentangling multiple types of recovery from brain injury. In J. Krieglstein (Eds), Pharmacology of Cerebral Ischemia (201216). Guildford: Medpharm. https://www.google.com/ books/edition/Pharmacology_of_Cerebral_Ischemia_2000/ Fj6Q4BXHs58C?hl=en
Smiałowska, M., Szewczyk, B., Woźniak, M., WawrzakWleciał, A., \& Domin, H. (2013). Glial degeneration as a model of depression. Pharmacological Reports, 65(6), 1572-1579. [DOI:10.1016/S1734-1140(13)71518-4] [PMID]

Sofroniew, M. V., \& Vinters, H. V. (2010). Astrocytes: Biology and pathology. Acta Neuropathologica, 119(1), 7-35. [DOI:10.1007/s00401-009-0619-8] [PMID] [PMCID]

Song, J. J., Oh, S. M., Kwon, O. C., Wulansari, N., Lee, H. S., \& Chang, M.Y., et al. (2018). Cografting astrocytes improves cell therapeutic outcomes in a parkinson's disease model. Journal of Clinical Investigation, 128(1), 463-482. [DOI:10.1172/ JC193924] [PMID] [PMCID]

Stavrovskaya, A. V., Yamshchikova, N. G., Ol'shanskiy, A. S. Konovalova, E. V., \& Illarioshkin, S. N. (2017). Transplantation of neuronal precursors derived from induced pluripotent stem cells into the striatum of rats with the toxin-induced model of huntington's disease. Human Physiology, 43(8), 881-885. [DOI:10.1134/S0362119717080114]

Takada, M., \& Hattori, T. (1986). Fine structural changes in the rat brain after local injections of gliotoxin, alpha-aminoadipic acid. Histology and Histopathology, 1(3), 271-275. [PMID]

Verkhratsky, A., Parpura, V., Pekna, M., Pekny, M., \& Sofroniew, M. (2014). Glia in the pathogenesis of neurodegenerative diseases. Biochemical Society Transactions, 42(5), 12911301. [DOI:10.1042/BST20140107] [PMID]

Villalba, R. M., Mathai, A., \& Smith, Y. (2015). Morphological changes of glutamatergic synapses in animal models of parkinson's disease. Frontiers in Neuroanatomy, 9(117). [DOI:10.3389/fnana.2015.00117] [PMID] [PMCID]

Voloboueva, L. A., Suh, S. W., Swanson, R. A., \& Giffard, R. G. (2007). Inhibition of mitochondrial function in astrocytes: Implications for neuroprotection. Journal of Neurochemistry, 102(4), 1383-1394. [DOI:10.1111/j.1471-4159.2007.4634.x] [PMID] [PMCID]

Watanabe, S., Fusa, K., Takada, K., Aono, Y., Saigusa, T., \& Koshikawa, N., et al. (2005). Effects of alpha-methyl-p-tyrosine on extracellular dopamine levels in the nucleus accumbens and the dorsal striatum of freely moving rats. Journal of Oral Science, 47(4), 185-190. [DOI:10.2334/josnusd.47.185] [PMID]

Wilhelmsson, U., Li, L., Pekna, M., Berthold, C. H., Blom, S., \& Eliasson, C., et al. (2004). Absence of glial fibrillary acidic protein and vimentin prevents hypertrophy of astrocytic processes and improves post-traumatic regeneration. Journal of Neuroscience, 24(21), 5016-5021. [DOI:10.1523/JNEUROSCI.0820-04.2004] [PMID] [PMCID]

Willoughby, J. O., Mackenzie, L., Broberg, M., Thoren, A. E. Medvedev, A., \& Sims, N. R., et al. (2003). Fluorocitrate-mediated astroglial dysfunction causes seizures. Journal of Neuroscience Research, 74(1), 160-166. [DOI:10.1002/jnr.10743] [PMID]

Zhao, Y., Keshiya, S., Atashrazm, F., Gao, J., Ittner, L. M., \& Alessi, D. R., et al. (2018). Nigrostriatal pathology with reduced astrocytes in LRRK2 S910/S935 phosphorylation deficient knockin mice. Neurobiology of Disease, 120, 76-87. [DOI:10.1016/j.nbd.2018.09.003] [PMID] [PMCID] 